\title{
Non-inferiority and equivalence trials
}

\author{
Ranstam J, Cook JA
}

Many randomised trials are designed to evaluate if the effect of one treatment differs (to any degree) from that of a comparator treatment; this type of study is known as a superiority trial. The comparator can either be medically effective or, with the use of placebo, ineffective. Where a new treatment is compared to a placebo (or minimal treatment i.e. active monitoring) this approach fits well. Placebo treatment comparator in particular are, however, not always ethical as the benefits, risks, burdens and effectiveness of a new treatment usually must be tested against those of an accepted treatment if it exist (1). A superiority approach also fits well with the scenario where two competing treatments are compared with the possibility of different responses. Superiority is typically assessed in terms of statistical evidence of a difference of any magnitude i.e. statistical superiority.

However, it is not usual that a new treatment is thought to have fewer side effects, fewer complication and lower costs but is at best hoped to have the same efficacy as an established treatment. In this context a superiority trial may be less useful as failure to (statistically) show superiority does not necessarily indicate clinical similarity. It can just reflect an inadequate sample size and consequentially a lack of precision in the statistical analysis.

A formal assessment of "non-inferiority" by using a non-inferiority trial design could be a better alternative. This study design requires a pre-defined non-inferiority margin for the primary outcome which is incorporated into the sample size calculation. The statistical analysis is based on estimating whether the new treatment is at least as efficacious as the comparator, which is defined as being within the magnitude of a non-inferiority (or if a two sided assessment, equivalence)_margin of the established treatment effect e.g. wound infection level within 5\%. The evaluation of non-inferiority trials as well as equivalence trials (the two-sided equivalence of a non-inferiority trial) and superiority trials can be based upon the corresponding confidence intervals, see Figure 1.

Non-inferiority and equivalence trials are more complex to implement and have several complications; the design, conduct, analysis, reporting and interpretation of these studies require special scrutiny in several respects (2-4). First, the pre-specification of the non-inferiority margin is crucial, and the definition of the margin needs to be based on both statistical and clinical considerations in order to ensure that the effect of the established treatment is sufficiently preserved. Second, the statistical analysis needs to reflect the design. A key difference is that the intention-to-treat (ITT) analysis, which in superiority trials is generally accepted as the preferred and unbiased approach, is not necessarily conservative in non-inferiority and equivalence trials (4). Third, the sample size calculation is more complex and is sensitive to minor differences in anticipated effects of the treatments. Fourth, if the confidence interval for the treatment difference excludes not only the non-inferiority (or equivalence) margin but also zero, it is adequate to consider the outcome of the non-inferiority trial as evidence for (statistical) superiority. The opposite approach, failing to show superiority in a superiority trial and instead claiming noninferiority (or equivalence), is not valid unless a pre-specified clinically important difference can be ruled out. 


\section{Reference}

1. Declaration of Helsinki World Medical Association. Available from: http://www.wma.net/e/ethicsunit/helsinki.htm [accessed on 15 July 2015].

2. EMA. Points to consider on switching between superiority and non-inferiority. European Medicines Agency, London, 27 July 2000, CPMP/EWP/482/99.

3. EMA. Guideline on the choice of non-inferiority margin. European Medcines Agency. London, 27 July 2005. EMEA/CPMP/EWP/2158/99.

4. Piaggio G, Elbourne DR, Altman DG, et al. Reporting of noninferiority and equivalence randomized trials. An extension of the CONSORT statement. JAMA. 2006;295:1152-1160.

5. Wiens BL, Zhao W. The role of intention to treat in analysis of noninferiority studies. Clinical Trials. 2007;4:286-291. doi: 10.1177/1740774507079443.

Word count: 357 
95\% Confidence Intervals and Trial Outcome

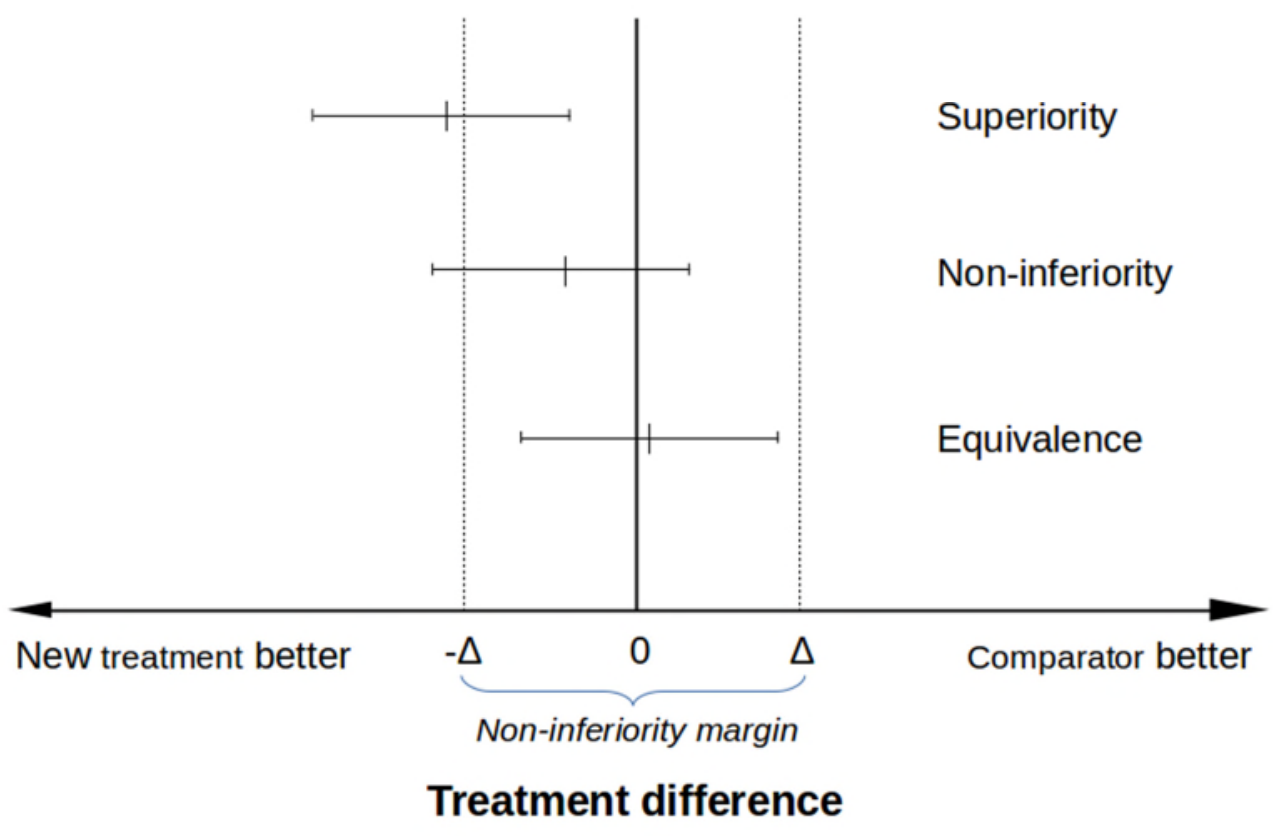

